\title{
Role of Radiation-induced TGF-beta Signaling in Cancer Therapy
}

\author{
Horatiu C. Dancea ${ }^{1}$, Mohammed M. Shareef ${ }^{2}$ and Mansoor M. Ahmed ${ }^{2^{*}}$ \\ ${ }^{1}$ Department of General Surgery, Geisinger Clinic, Danville, Pennsylvania. ${ }^{2}$ Department of \\ Radiation Oncology, Sylvester Comprehensive Cancer Center, Miller School of Medicine, \\ University of Miami, Miami, Florida
}

\begin{abstract}
TGF- $\beta$ signaling regulates several different biological processes involving cell-growth, differentiation, apoptosis, motility, angiogenesis, epithelial mesenchymal transition and extracellular matrix production that affects embryonic development and pathogenesis of various diseases, including cancer, its effects depending on the cellular context and physiological environment. Growth suppression mediated by TGF- $\beta$ signaling often associated with inhibition of c-myc, cdks and induction of p15, p27, Bax and p21. Despite its growth inhibitory effect, in certain conditions TGF $-\beta$ may act as a promoter of cell proliferation and invasion. Loss of responsiveness to growth suppression by TGF- $\beta$ due to mutation or loss of TGF-beta type II receptor (TRRII) and Smad4 in several different cancer cells are reported. In addition, TGF- $\beta$ binding to its receptor activates many non-canonical signaling pathways. Radiation induced TGF- $\beta$ is primarily involved in normal tissue injury and fibrosis. Seminal studies from our group have used radio-adjuvant therapies, involving classical components of the pathway such as TRRII and SMAD4 to overcome the growth promoting effects of TGF- $\beta$. The main impediment in the radiation-induced TGF- $\beta$ signaling is the induction of SMAD7 that blocks TGF- $\beta$ signaling in a negative feedback manner. It is well demonstrated from our studies that the use of neutralizing antibodies against TGF- $\beta$ can render a robust radio-resistant effect. Thus, understanding the functional interactions of TGF- $\beta$ signaling components of the pathway with other molecules may help tailor appropriate adjuvant radiotherapeutic strategies for treatment of solid tumors.
\end{abstract}

Received 12/19/08; accepted 02/03/09

${ }^{*}$ Correspondence: Mansoor M. Ahmed Ph.D., Department of Radiation Oncology, Sylvester Comprehensive Cancer Center, Miller School of Medicine, University of Miami, 1475 NW 12 ${ }^{\text {th }}$ Avenue Suite 1500 (D-31), Miami, FL 33136, USA. Tel. 305.243.5454, Fax. 305.243-1854; email: amansoor@med.miami.edu
Keywords: TGF- $\beta$; Smads; Radiation; Cancer; Apoptosis

\section{Introduction}

Cell proliferation is a very intricate and complex process involving both stimulatory and inhibitory signals. Abnormal proliferation observed in cancer cells is caused by mutations that either increase positive growth signals or decrease negative growth control signals or a combination of both events. TGF$B$ signaling is one such regulatory signal that is manipulated during abnormal proliferation seen in several tumors such as pancreatic, breast and colon. While the role of TGF- $B$ in wound healing is extensively investigated field of research, effect of TGF- $\beta$ on tumor suppression and progression has received very little attention, with significant developments occurring during the last few decades. TGF- $B$ demonstrates both tumor-suppressive effects that malignant cells must overcome to continue their development and oncogenic effects stimulating cell proliferation and invasion once the suppressor activity is inhibited. TGF- $B$ regulates processes such as immune regulation and cell invasion, as well as microenvironment modifications that malignant cells may use to their advantage (1). It is generally agreed that in early stages, TGF- $B$ plays an important role in suppression of malignancy and at later stages in tumor progression. TGF- $B$ is also an important modulator of immunity, particularly malignant cells that elude from growth suppressive effects are able to produce large amounts of this cytokine and thus are protected against immune response. In normal or premalignant cells, TGF- $B$ inhibits tumor progression directly through cellautonomous tumor-suppressive effects (cytostasis, differentiation, apoptosis) or indirectly through its effects on the stroma (suppression of inflammation and stroma-derived mitogens). When growthsuppressive function is lost in malignant cells, TGF- 
B pathway is used to initiate immune evasion, to produce growth factors, to differentiate into a more invasive phenotype and facilitate dissemination leading to metastasis. While, the molecular event leading to this switch from tumor suppressing role to tumor promoting, has remained elusive, studies have suggested that host immune cells may play a role in this transformation (2).

Regulation of TGF- $B$ signaling becomes more complicated with the interaction of TGF- $B$ signaling components with members of other signaling pathways and non-canonical TGF- $B$ pathways. In addition regulation of TGF- $B$ signaling by the cellular microenvironment such as hypoxia has been observed in our studies. Yet another layer of complexity is added to the process of tumorigenesis by the selective up regulation of specific miRNA clusters. In gastric cancer, miR-106b and miR-93 were shown to suppress p21 expression, miR-25 silences pro-apoptotic gene BCL2L11 (BIM) expression, and these genes are essential for TGF- $B$ mediated cell cycle arrest and apoptosis respectively (3).

Although, radiation induced TGF- $B$ was implicated in radiation-induced fibrosis and normal tissue injury (4), the role of radiation-induced TGF- $B$ in tumor suppression remains largely unexplored, due to intrinsic deficiencies of this pathway in many cancer cells. Adjuvant gene therapeutic agents, including some against the specific miRNA sequences, can be combined to exploit the useful effects of radiation induced TGF- $B$ in controlling the tumor. This review largely focuses on such novel therapeutic approaches.

\section{TGF-beta pathway: a canonical overview}

The human TGF- 6 family is constituted by more than 30 factors separated into two groups: one contains factors like TGF-B, activin, myostatin, nodal and lefty, while other group comprises of bone morphogenic proteins (BMP), anti-mullerian hormone (AMH) and other growth and differentiation factors. Many of these factors in the second group are key regulators of embryonic stem cell differentiation, body axis formation and organogenesis, while role in adults is related to gonadal regulation by activin, inhibition of muscle development by myostatin bone growth and repair by BMPs (5). TGF-B's are made as preproteins that are cleaved to form a homodimer of two polypeptides connected by a disulfide bond. TGF- $B$ molecule exists in 3 isoforms: TGF- $81, B 2$ and 83 . TGF- 81 is found predominantly in the immune system (6). The complex of TGF-81, Latency Associated Protein (LAP, derived from propeptide), Latent TGF- $B$ binding protein (LTBP) is called as large latent complex (LLC) which is activated by several different molecules such as proteases, thrombospondin- 1 , reactive oxygen species, and the integrins such as avB6 (7). The complex of TGF-81 and LAP is referred to as small latent complex (SLC). LTBP1 plays a critical role in avB6-mediated latent TGF- $B$ activation and enhancement of SLC secretion (8). It is interesting to note that radiation induces integrin avb6 in lungs (9). TGF- 6 signals by binding to transmembrane serine-threonine kinases receptors, initially with type II receptor (RII); this receptor-ligand complex recruits and homodimerizes with RI by phosphorylation of the glycine-serine-rich domain resulting in activated type I receptor (RI) that serves as a docking site for Smad 2 and Smad 3 proteins. A third receptor (RIII), although not believed to be involved directly in TGF- $B$ signaling, acts to present TGF- $B$ to RII. The receptor complex consists of two types, type II and type I receptors. Upon phosphorylation by RI, the Smad 2 and Smad 3 , dissociates from RI and are commonly referred as receptor activated Smads (RSmads). At RI, the ISmads, Smad6 and 7, act as competitive inhibitors of R-Smad phosphorylation. SARA (Smad Anchor for Receptor Activation) and cPML (cytoplasmic Promyelocytic Leukemia Protein) recruit Smad2 and Smad3 for phosphorylation by the TGF- $B$ receptor. The cPML protein is sequestered in the nucleus by TGIF (TGF-beta-Interacting Factor) thereby negatively regulating TGF- $B$ signaling pathway. PCTA (PML competitor for TGIF association) has been shown to compete with cPML for binding to TGIF, thereby promoting TGF- 8 signaling. (10). One or both RSmad's associates with Smad 4, also called co-Smad, forming a heterodimeric, heterotrimeric or heterotetrameric complex, translocates to the nucleus, where it associates with other transcriptional co-activators (11). When in basal state Smad's may shift in and out of the nucleus, but once phosphorylated by RI, RSmads will accumulate in the nucleus. Once the TGF- $B$ signaling is terminated, RSmads are dephosphorylated rapidly and exported to cytoplasm. Multiple Smads have been reported of which Smad 2 and 3 act as signal transducers for TGF- $B$ pathway, while Smad 1, 5 and 8 mediate signals for BMP-like ligands (1) (Fig. 1). The DNA-binding properties of $\mathrm{R}$-Smads and $\mathrm{Co}^{-}$ Smad have been extensively analyzed, and the minimal Smad binding element (SBE) contains only four base pairs, 5'-AGAC-3', called the CAGA 


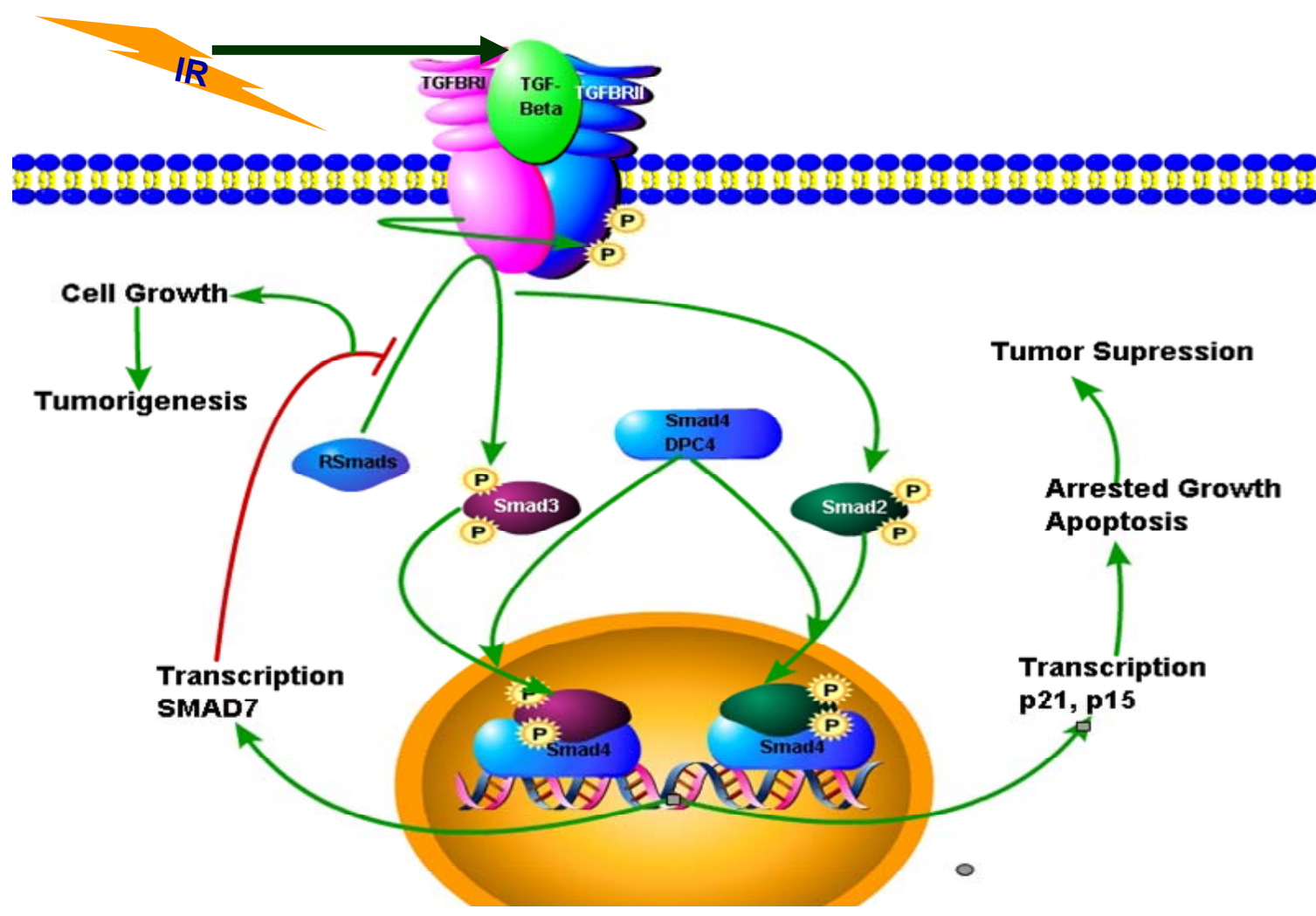

Figure 1. Dual effects of TGF- $\beta$ signaling on the growth of cells and impact of radiation. Classical canonical TGF- $\beta$ signaling pathway showing the dual effects of this signaling on the cell growth. Normally this pathway induces cell growth suppressive effects, however, during the process of tumorigenesis due to several factors TGF- $\beta$ signaling pathway induces proliferation, invasiveness, angiogenesis, metastasis and immune suppression. In this figure, based on the findings from our group, radiation can potently induce TGF- $\beta$ and this TGF- $\beta$ can be activated to exert negative growth effects by inducing the expression of cdk inhibitors such as p21 and p16. It is also shown here that the inhibitory Smad7 can be induced by Smad4 that can in turn inhibit the TGF- $\beta$ signaling.

box (12). Understanding the canonical TGF- $B$ signaling from a canonical view subjugated Smads to the function of mere transducers of this pathway. However this pathway is far more complex than what the canonical view (Fig. 1) has proposed.

\section{TGF-beta pathway: a non-canonical overview}

A simple scheme of ligand to receptor to activated transcription factor is highly regulated and riddled with crosstalk from other pathways. Inspite of DNA binding ability of Smads, the Smad4RSmad complexes must associate with additional DNA-binding cofactors in order to achieve binding with high affinity and selectivity to specific target genes. These Smad partners are drawn from various families of transcription factors, including forkhead, homeobox, zinc-finger, bHLH, AP1 families and others $(13,14)$. Each Smad4-RSmad-cofactor combination is determined by the presence of transcription factor specific cognate binding sequence element in the regulatory regions of target genes. Activated Smad complexes additionally to recruit transcriptional co-activators, co-repressors and chromatin remodeling factors that may ultimately lead to stimulus which can activate or repress hundreds of target genes at once. These transcriptional co-activators increase transcription by bringing the sequence-specific transcription factors in close proximity to RNA polymerase II complex. CBP/p300 function requires presence of Smad 4 to stabilize the interaction between RSmad with CBP (15). Tang et al., described a noncanonical role for I-Smad 7, where they show that SMAD7 directly interacts with B-catenin and inhibits its phosphorylation by glycogen synthase kinase 36 (GSK-3B) and casein kinase Ia (CKIa), resulting in decreased $B$-catenin degradation (16). Non-canonical functions for R-Smads (Smad1, 2 and 3) have also been described which include posttranscriptional processing of miR-21 (17). The miR21 down-regulates the tumor suppressor PDCD4 (Programmed Cell Death 4) suggesting that Smads as promoters of tumorigenesis $(18,19)$. However, it is important to note that phosphorylation status of 
the Smads does not affect the miRNA processing activity of Smads.

Phosphorylation of R-Smads on S-S-X-S (where $\mathrm{X}$ is any amino acid) motif can be achieved by factors and kinases other than TGF- $B$ and TBRI respectively. Epidermal Growth Factor (EGF) and Hepatocyte Growth Factor (HGF) induce R-Smad phosphorylation although the mechanistic details such as the kinase responsible for this function are not characterized. In addition to these studies, Mps1, a kinase involved in cell cycle and mitotic control and activated by a microtubule destabilizing agent, nocodazole, has been shown to be phosphorylate RSmads in a TGF- $B$ independent manner (20)

\section{Crosstalk between TGF-ßeta and other signaling pathways}

The complexity of crosstalk between TGF- $B$ and other signaling pathways are overwhelmingly intricate and highly context dependent. We present some of the ways TGF- $B$ signaling could be manipulated.

\section{MAPK and TGF- $\beta$ pathway}

Her2/Neu/ErbB2 signaling activated both MAPK and PI3K/Akt pathways and interacts with TGF- $B$ signaling pathway in breast cancer. A general opinion emerging from these studies is that Her2/Ras can antagonize TGF-B-induced apoptosis and cell cycle arrest, while metastatic function of TGFB is unchecked (21-23). Both negative and positive regulations exist between these pathways. While JNK kinases seem to negatively regulate autocrine TGF- $B$ expression, MEK/Erk has been reported to positively regulate SMAD3 expression in epithelial and smooth muscle cells $(24,25)$. Erk mediated phosphorylation of linker region of SMAD3 is known to inhibit its transcriptional activity but does not affect its nuclear localization (26). On the other hand phosphorylation by other kinases including Rho-dependent Kinase (ROCK) and p38MAPK at serine203/207, facilitates rather than inhibit TGF-8-mediated growth inhibition (27). Oncogenic Ras, along with JNK and p38 phosphorylate SMAD4 or mutant SMAD4 reduces the protein stability of Smad4 (28) Further these kinases have a role in transcriptional regulation of SMAD7, indirectly regulating TGF-B signaling. (29, 30).

\section{PI3K/Akt and TGF- $\beta$ pathways}

PI3K/Akt signaling abrogates the pro-apoptotic function of TGF- $B$ in response to insulin, IGF, IL-6 and some viral proteins (31-34) with Smad3 but not Smad2 as the primary target of inhibition by PI3K/Akt pathway. How PI3K/Akt mediates inhibition of Smad3 remains to be answered.

\section{Wnt and TGF- $\beta$ pathways}

A large number of shared target genes of Wnt and TGF- $B$ pathway are regulated by $\mathrm{Smad} / \mathrm{B}^{-}$ catenin/Lef protein complex in a synergistic manner. In addition mutual regulation of TGF- $B$ and Wnt ligands is important during development as well as in adult tissues (35).

\section{Interaction of rest of the signaling pathways with TGF- $\beta$ pathway}

Sonic Hedgehog (Shh) induces an invasive phenotype of cultured gastric cancer cells, which is thought to be mediated by Shh-mediated expression of TGF- $B$ ligand and TBRI (36). While the interaction of Shh pathway with TGF- $B$ has been extensively shown in development of drosophila and zebra fish, the role of this interaction in the process of carcinogenesis needs to be examined. There is extensive crosstalk of TGF- $B$ and Notch signaling that could affect several functions of TGF- $B$ signaling such as epithelial mesenchymal transtion (EMT). Smad3 mediated expression of Jagged 1 and Hey1 is necessary for TGF- $B$-induced EMT (37). Jagged 1 up regulation contributes to TGF- $B$ stimulated p21 expression and cytostasis in epithelial cells (38). Smad3 and NICD (Notch Intracellular Domain) directly interact with CSL to form a specific DNA-binding complex at the promoter of Hes 1 (39). Activated Notch 1 inhibits the TGF- $B$ mediated pro-apoptotic function by sequestering the p300 co-factor from Smads (40).

The function of Interleukins, TNFa, and IFNY and their bioavailability is modulated by TGFB and vice versa. Most of these factors are key regulators of immune functions and inflammatory responses. The role of these interactions in the process of tumorigenesis has received much less attention.

Thus, there is no simple rule to describe how TGF- $B$ interacts with other signaling pathways. The main concept is TGF- $B$ signaling crosstalk is context dependent, differentially occurring with parameters such as cell type, developmental stage, physiological, pathological, microenvironment, protein localization, modifying enzymes, co-factors, targets among several other variables. The outcome of all crosstalks between several different pathways is an integrated reflection of the state of the cell. Identifying the exact defects in these integrated circuits during the process of tumorigenesis is a 


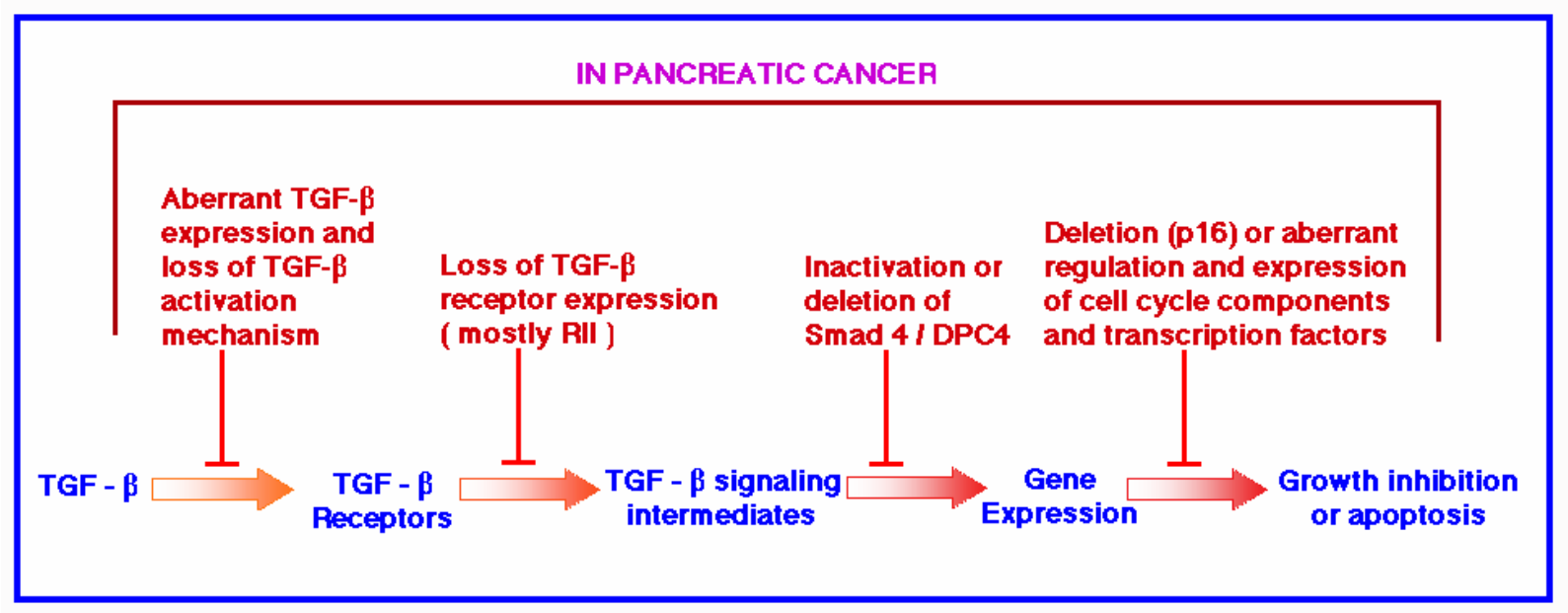

Figure 2. A schematic representation of genetic lesions at different stages of TGF- $\beta$ that lead to tumorigenesis in pancreatic cancer model. In a normal condition, TGF- $\beta$ signaling leads to growth suppression. However due to mutations or loss of several key genes involved in this signaling pathways, the growth suppressive effects are transformed to growth proliferative effects. SMAD4 and T $\beta R I I$ genes are often mutated in pancreatic carcinoma. Alteration in any of these components can lead to abnormal cell proliferation and render radio-resistance phenotype.

challenging task that needs to be rectified by making enough changes to tilt the scale in favor of effective tumor control. Adding radiation to this scheme of things adds another layer of complexity to the process of tumor control. TGF- $B$ pathway responses in addition to its crosstalk with radiationinduced signaling network needs to be analyzed for effective use of radiation in tumor control.

\section{TGF-ßeta signaling pathway and malignancy}

TGF-Bs elicit their effects by binding to cell surface receptors. The tumor suppressor activity of TGF- $B$ in predominantly mediated through its effect on cell cycle progression and apoptosis. Inhibition of c-Myc, which is known to promote cell cycle entry into $\mathrm{S}$ phase by regulating various cell cycle related genes, by TGF- $B$ signaling is very well established (41-43). Repression complex of SMAD3, E2F4/5 and p107 which are induced upon TGF- $B$ signaling repress the expression of c-Myc (44). Stimulation of $\mathrm{p} 15^{\text {ink } 4 \mathrm{~b}}$ and $\mathrm{p} 21^{\text {cip } 1}$ expression by TGF- 6 leads to inhibition of cyclin D-CDK4/6 and Cyclin A/E-CDK2 activity $(45,46)$. The mechanisms by which TGF- 8 induces apoptosis are varied and include induction of caspases, down-regulation of $\mathrm{Bcl}-\mathrm{X}, \mathrm{Bcl}-2$ and cyclin $\mathrm{D} 1$, inhibition of c-myc, cdks, cdk4-associated $\mathrm{Rb}$ kinase activity; induction of cdk inhibitors p15 and p27; induction and activation of Bax (47-50). Our recent findings suggest that TGF- $B$ directly regulates the expression of Bax through the Smad binding element (SBE) ${ }^{1}$,

Studies have shown that a variety of carcinoma cells lose their growth inhibitory response to TGF- $B$ (51-55). In fact tumors secrete copious amount of activated TGF- $B$ which is thought to facilitate invasion and metastasis, angiogenesis, and suppression of antitumor immune responses (56) (Fig. 1). Escape from TGF- 8 's negative growth regulation thus provides cells with a selective growth advantage and accelerates tumor progression. Since the heteromeric complex of RI and RII is essential for TGF- $B$ signaling, it has been suggested that loss of these receptors could cause TGF-beta insensitivity and thus contribute to uncontrolled growth of cells. A study on the pancreatic cancer cell line MIA $\mathrm{PaCa}^{-2}$ demonstrates resistance to TGF- $B$ - induced growth inhibition due to a lack of RII gene expression (57). Growth inhibitory effect of TGF- $B$ was restored after expression of RII cDNA in a mutant hepatoma cell line, Hep 3B-TR, that lacks a functional RII (58). Similarly, expression of RII cDNA led to reduced malignancy in the human breast cancer cell line, MCF-7 (51). Studies of HNPCC suggest that mutations in RII may result from micro-satellite instability (59). Further reports in a variety of

\footnotetext{
${ }^{1}$ Reeves et al. Smad-4-mediated up-regulation of Bax is essential in eliciting radiation-induced apoptotic response in pancreatic adenocarcinoma. Manuscript in preparation.
} 
carcinoma cell lines $(60,61)$ also provide evidence of an association between MIN and mutations in RII, and a possibility that RII is a tumor suppressor gene (62). The mutations found in RII were 1-2 bp insertions or deletions in the simple repeats of coding sequence and resulted in non-functional RII transcripts. Missense mutations in the kinase domain of the RII gene resulted in loss of functional receptors in head and neck squamous carcinoma cell lines (63). These studies support the premise that structural defects in RII would be central to disabling ligand binding and complex formation, hence inhibiting TGF- $\beta$-mediated signal transduction. However, in contrast to HNPCC, we failed to observe a significant number of mutations in simple repeat sequences of the RII gene in pancreatic adenocarcinoma (64). In addition, deletion or intragenic mutations of Smad-4 (DPC4), a tumor suppressor gene located on chromosome 18, can cause a loss in response to TGF- 6 (65).

The Smad-4 gene is reported to be homozygously deleted in about $30 \%$ of pancreatic cancer and to be inactivated by intragenic mutations in another $20 \%$ of these cancers (66). We found that most of the pancreatic cancer cell lines showed no growth inhibitory response to exogenously supplied TGF- $B$. Since RII was not often mutated in pancreatic cancer, we anticipated that downregulation of TGF- $B$ RII receptors might be responsible for the loss of response to TGF- $B$ induced growth inhibition. Intragenic mutations or loss of expression of Smad-4, an essential down-stream target of TGF- $B$ signaling pathway could cause a similar loss in response to TGF-8. Our earlier findings revealed that most pancreatic cancer cell lines showed down regulation of the mRNA expression of TGF- $B$ receptors and Smad-4 (67). On the contrary, few reports from Dr Korc's lab showed up-regulation of RII mRNA when compared to the expression in normal pancreas. This upregulation of RII receptor correlated with poor prognosis (68, 69). Interestingly, the DNR transgenic mice (overexpressing dominant-negative mutant for RII to block the endogenous function of RII) showed increased proliferation of pancreatic acinar cells and severely perturbed acinar differentiation $(70,71)$. These findings strongly suggest that dysfunction in RII is an important indicator of pancreatitis and pancreas cancer.

In addition to the loss or mutation of RII and SMAD4 genes, deletion or inactivation of TGF- $B$ effector genes such as p16 (MTS1) is also observed (72). Genetic studies in mice have provided evidence for TGF- 6 in tumor suppression. TGF-B1 hetorozygous null mice show increased liver cell proliferation with reduced apoptosis and high incidence of tumor formation when challenged with carcinogens (73). Homozygous loss of SMAD2 and SMAD4 lead to death of mice in utero, their heterozygous null counterparts are viable, with SMAD4 heterozygous mice develop gastric polyps that can form tumors at a later age $(74,75)$. Although no mutations for SMAD3 have been reported so far, mice with homozygous deletion for SMAD3 gene develop aggressive colorectal cancer. In addition to the classical targets several new targets such as ELF, a B-spectrin, which plays a crucial role in the propagation of TGF- $B$ signaling (76) have been identified. ELF associates with Smad 3 and the TGF- 6 receptor complex; leading to further interaction with Smad4 and their translocation to the nucleus. Mis-colocalization of Smad3 and Smad4 were observed in mice null homozygous for ELF causing disruption of TGF- $B$ signaling (77). Loss of TGF- $B$ signaling through $B^{-}$ spectrin ELF leads to hepatocellular cancer through cyclin D1 activation (78). Thus, it appears that TGF$B$ signaling could be affected through disruption of several different components, some characterized, others needing more investigation. Further, other signaling pathways, may affect the outcome of TGF$B$ pathway. A schematic representation of multiple targets that get affected in pancreatic cancer cells leading to the loss of growth suppressive affects of TGF-B signaling is shown in Fig. 2.

\section{Radiation-induced TGF-beta signaling}

TGF- $B$ was found in relation to the pathological changes of late radiation damage in the non-tumorbearing tissues of previously irradiated patients, and thus it is well recognized that TGF- $\beta$ activity may modulate late post-radiation changes (79). While IR was shown to specifically induce expression of TGF81 isoform, it down regulates the TGF- 83 isoform and does not influence the TGF-B2 isoform $(80,81)$. All this responses are required for post-irradiation DNA repair, progression through cell cycle, inflammation in an early stage, and later development of radiation damage such as fibrosis. Also, the TGF- 81 type I promoter was significantly increased at 6 hours after radiation, showing that promoter activation is indeed mediated by irradiation. It was well documented that the fibrosis formation following radiotherapy results not only from TGF- $\beta$ produced locally in the injured normal tissue, but also from circulating TGF- $\beta$ released by the tumor. Thus, radiotherapy-induced normal 


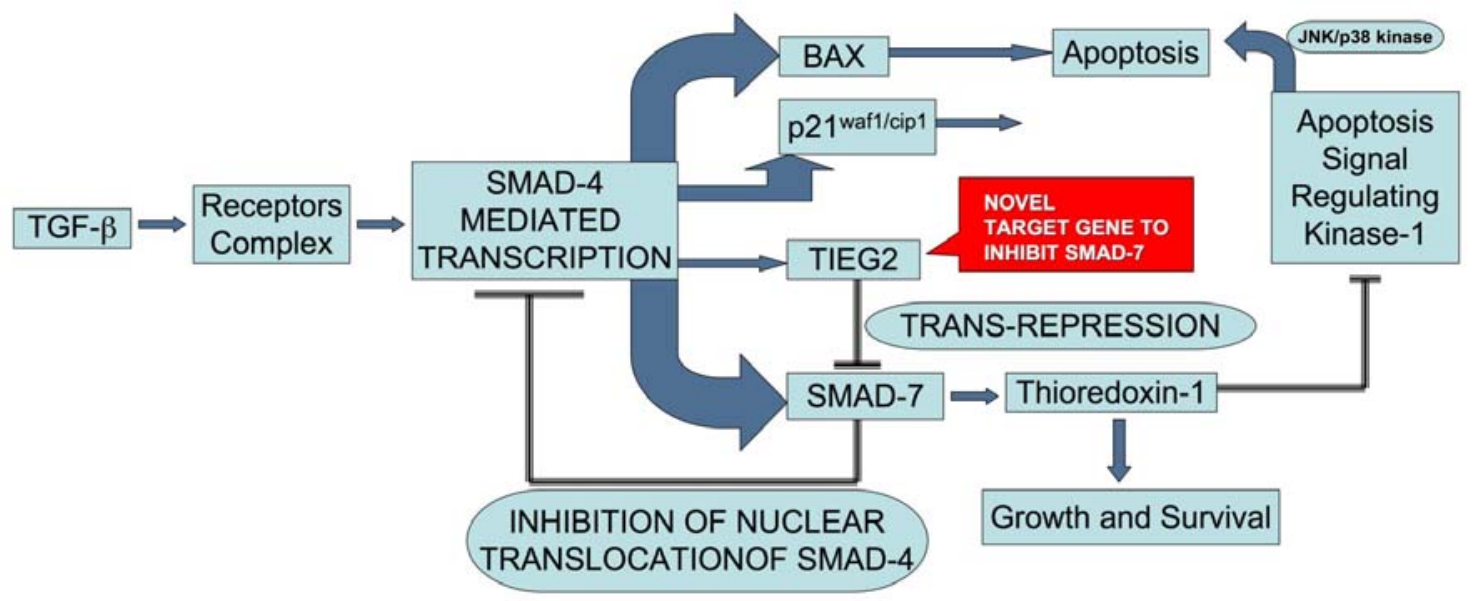

Figure 3. Radiation induced SMAD7, a bottleneck in radiotherapy. As a negative feedback mechanism, radiation-induced TGF- $\beta$, elevates the expression of SMAD7, which blocks the activity of this pathway. TIEG2 (also known as KLF11) is a tumor suppressor gene that causes repression of SMAD7 expression and can be a useful target for adjuvant radiotherapy to harness the negative growth effects of TGF- $\beta$ signaling pathway. Thus, over expression of TIEG2 will drive the TGF- $\beta$ pathway towards the growth suppressive functions and enhance the radiation response.

tissue damage appears in part to be a local manifestation of a systemic condition (82).

There is extensive evidence on the critical role played by TGF- $B$ in the initiation, development, and persistence of radiation-induced fibrosis. Current trend focuses to develop agents that can specifically target TGF- 8 pathway to inhibit radiation-induced fibrosis of the normal tissues (4) and one such agent is to use the soluble-form of TGF- $B$ type II receptor that can bind TGF- 8 in a dominant-negative fashion, and then consequently block the TGF- $\beta$ function (83). To further support this notion in a clinical situation, in a screen of serum samples from patients treated with high-dose radiation that was delivered through GRID, we found that TGF- $B$ was not elevated and failed to correlate with tumor regression response. And, these patients did not slow any clinical symptoms of fibrosis or normal tissue injury (84). Thus, this is another example where inhibiting the elevation of TGF- $B$ in response radiation can mitigate normal tissue injury and abrogate fibrosis.

The serum levels of TGF- $B$ in certain patients may potentially dictate tumor response to radiotherapy. Lower pretreatment TGF- $B$ and VEGF levels were found to be associated with tumor response to chemoradiation (85). In an in-vitro study, the levels of the active form and total TGF- 6 in the supernatants from irradiated malignant glioma cells was found to be decreased compared to those from un-irradiated cells. However, since irradiation inhibited the growth of malignant glioma cells, the amount of TGF- $B$ secretion per cell in irradiated cell

compartment was found to be elevated after irradiation. These results suggest that malignant glioma cells can still secrete TGF- $B$ and activate latent TGF- $B$ even after large dose irradiation, despite the inhibition of tumor growth (86). These observations raise the questions whether the activated form TGF- $B$ induced in response to radiation can participate in the growth inhibition of tumor cells provided these tumor cells harbor intact components of TGF- $B$ downstream signaling pathway.

To understand the above posed questions, our group initiated several studies with the hypothesis that the radiation induced TGF- $B$ will be involved primarily in negative growth regulation and cell death. We previously reported that radiation induces an endogenous TGF- $B$ protein that exerts clonogenic inhibition and cause apoptosis in tumor cells that harbor intact TGF-B signaling pathway. The absence or low level of expression of RII or Smad4 is thought to be reason for resistance to chemo- or radiotherapy in lung cancer. We showed that restoration of RII receptor in normal lung cells that specifically harbored defective TGF- $\beta$ signaling pathway increased sensitivity to radiation and will decrease malignant potential by increasing apoptosis (87). The wild-type p53 protein is reported to affect radiation responsiveness in several types of cancers, with radiation causing an accumulation of 
p53 protein that initiates apoptosis. p53 was found to be mutated and nonfunctional in the majority of pancreatic tumors, and this mutation is recognized as an important factor in poor prognosis of this disease playing an important role in the resistance of cancers to radiation and chemotherapy.

Our studies show that abrogation of endogenous TGF- $B$ function causes increased proliferative potential, as well as increased resistance to radiation-induced clonogenic inhibition and further rendered resistance to recombinant TGF- $B$ induced or radiation-induced apoptosis. Restoration of TGF- $B$ signaling pathway in p53 deficient pancreatic cancer cell line MIA PaCa-2 led to IR induced TGF- $B$ signaling, up-regulation of $\mathrm{p} 21$ and Bax activation caspases and enhanced sensitivity to IR (47). In addition, primary mouse embryonic fibroblast (MEF) transfected with a dominant negative TBRII mutant resulted in abrogation of radiation induced TGF- 6 responsive promoter activity with enhanced resistance to radiation-induced apoptosis.

TGF-61 plays a central role of activation of $\mathrm{p} 53$ and ATM signaling pathways, increasing apoptosis in irradiated cells. Whether the fast stimulation of p53 and ATM involves interaction between TGF- 61 RI-Smad signaling is still a field of controversy, as p53 and ATM are phosphorylated even when RI is inhibited (88). Low dose radiation (50 $\mathrm{mGy} \mathrm{Y}^{-}$rays) exposure of normal cells was found to have bystander effects on transformed cells leading to their apoptosis in a TGF- 6 dependent manner (89). In MCF-7 cell line resistant to Doxorubicin chemotherapy, a decrease in expression of TGF- $B$ receptors type I and II was observed, thus leading to resistance to radiation therapy as well. This refractoriness is caused at least partially by defective TGF- $B$ signaling pathway (90).

Activated Ras, often seen in carcinomas of the pancreas and colon, deregulates TGF- $B$ signaling by altering the expression of TGF- $\beta$ type II receptor (RII); tumors harboring mutant Ras are more resistant to radiation than cells with wild-type Ras (91-94). In addition, Ras inhibits TGF-B antiproliferative effect through suppression of Smad 2 or 3 nuclear translocation (95).

The protein p21 expression is induced by p53 after DNA damage or by TGF- $B$ signaling through the Smad pathway leading to G1 cell cycle arrest. In BxPC3 and Hs766T, with inactivated p53 and TGF- $B$ pathways (both cell lines have homozygous deletion of DPC4), radiation failed to induce an up-regulation of p21 protein, while DPC4 (Smad4) re-expression resulted in p21 protein elevation via direct transactivation of the $\mathrm{p} 21$ promoter in response to radiation-induced TGF- $B$ exposure ${ }^{1}$. Re-expression of functional RII receptor in MIA $\mathrm{PaCa}^{-2}$ cells enhanced TGF- $B$ mediated growth inhibition caused by both recombinant TGF- $B$ and radiation. This enhanced radiation sensitivity was associated with induction of Bax mRNA and release of cytochrome C and activation of caspase-3, suggesting a clear induction of programmed cell death. Bax is induced both by p53 and by TGF- 81 . Radiation failed to cause an increase in the expression of Bax protein in parental MIA PaCa-2 cells because both the p53 and TGF- 6 pathways are not functional, but restoring TGF- $B$ signaling led to Bax protein elevation after irradiation despite a nonfunctional p53 pathway, suggesting a clear induction of programmed cell death (47). Our recent data using Bax promoter constructs clearly shows a direct regulation of Bax by radiation-induced TGF- $B$ signaling ${ }^{1}$.

Restoration of TGF- 6 signaling is necessary to overcome the effects of a nonfunctional p53 pathway and can be the basis of the radiation sensitivity in these cells. Based on previous studies on eight human colorectal cancers, it was postulated that radiation-induced sensitization to TGF- $B$ require functional p53, but our study demonstrated that MIA PaCa-2/RII cells with restored TGF- 6 signaling are sensitive to radiation despite the fact that these cells harbor a mutated p53 gene (47). Importantly, radiation failed to induce p21 protein expression in parental MIA PaCa cells, since both the p53 and TGF- $B$ pathways are not functional in these cells, but after restoration of functional RII, radiation exposure resulted in p21 protein elevation (87).

Inhibiting Ras oncogenic activity may increase the sensitivity of tumor cells to radiation. Posttranslational activation of Ras is initiated by farnesyltransferase (FT), resulting in the prenylation of the Ras protein that binds to the membrane and induces transformation and proliferation. Based on this, we demonstrated that farnesyltransferase inhibitor (FTI) directly restores TGF-8 signaling through restoring the RII expression leading to induction of radiation sensitivity. Knowing that MIA PaCa-2 cells are highly resistant to exogenous recombinant TGF-81 protein (57), lack RII expression and devoid of TGFB signaling (67) with increasingly sensitive to FTI (L-744,832), we demonstrated that in MIA PaCa-2 cells FTI alone or FTI plus radiation caused an induction of RII expression. In these cells FTI induced an increased TGF- $B$ promoter activity, with an even more significant induction of activity in cells 
treated with both radiation and FTI, resulting in a parallel increase in apoptosis induction, indicating that FTI restores radiation-induced TGF- $B$ signaling by restoring RII expression and enhances the effect of radiation. This mechanism of effect of FTI was found to be at the promoter level to regulate the transcription of both RII and p21 induction. This activity was significantly inhibited by the presence of anti-TGF- $B$ neutralizing antibody. It was shown that FTI inhibited growth in five pancreatic cancer cell lines and this inhibition was associated with post-translational processing of $\mathrm{H}$-Ras and $\mathrm{N}$-Ras, but not K-Ras. (96). In a more recent study it was actually shown that irrespective of K-Ras mutational status, FTI was found to inhibit the farnesylation of Ras protein in pancreatic adenocarcinoma cells. This study showed inhibition of Ras activity after FTI treatment in two pancreatic cancer cell lines, one with wild-type K-Ras (BxPC-3) and the other with mutant K-Ras (MIA PaCa-2).

It was also reported that high levels of DNA methyltransferase 1 (dnmt1) levels are required to maintain Ras-transformed phenotype, indicating that dnmt1 is an important down stream effector of Ras oncogenic pathway. High levels of dnmt1 activity were found in many tumor cells in-vitro and in-vivo, and activation of oncogenic Ras signaling resulted in the induction of dnmt1 expression. Dnmt's are associated with methylation of several promoters and thus resulting in down-regulation of RII expression due to its promoter hypermethylation. Again, it was demonstrated that re-expression of RII by FTI or FTI plus radiation caused significant inhibition of the expression of dnmt1 when compared to the cells that were not treated by FTI. FTI treatment restored the RII expression through inhibition of dnmt1 levels causing elevation of functional TGF- $B$ signaling by radiation, suggesting a causal relation between the down-regulation of dnmt1 and upregulation of RII expression in response to FTI treatment. In summary, FTI mediated inhibition of dnmt1 expression resulted in the restoration of TGF- $B$ signaling via $\mathrm{RII}$ reexpression is a potential sensitizer to the effects of ionizing radiation (97).

As mentioned before, measuring the expression of the p21, one of TGF- 8 target genes and also inducible by p53, has been thoroughly studied (98). We also mentioned that MIA $\mathrm{PaCa}-2$ cells contain only one allele of p53 and this allele is mutated. When studied in MIA PaCa-2 cells p21 was upregulated cells treated with FTI, but not in cells treated with radiation alone, suggesting that the lack of up-regulation of p21 protein in irradiated MIA PaCa-2 cells may be due to dysregulation in both the p53 and TGF- 6 pathways. In MIA PaCa-2 cells treated with FTI, in the absence of functional $\mathrm{p} 53$, induction of RII expression and the restoration of TGF- $\beta$ signaling are the likely causes for the upregulation of $\mathrm{p} 21$ (97).

In addition to TBRII, mutations of Deleted in Pancreatic Carcinoma 4, DPC4 (SMAD4) is also frequently observed. $\mathrm{BxPC}-3$ cells with restored DPC-4 expression (by adenovirus) were significantly sensitive radiation-induced clonogenic inhibition when compared to cells over expressing adenoviral vector alone. Interestingly, bax protein was found elevated in the untreated samples and irradiated BxPC-3 cells overexpressing DPC-4. TGF-B signaling was found to directly regulate Bax by binding to SMAD binding element in the promoter of Bax. Similar findings were observed in another DPC-4 null pancreatic cancer cell line Hs667T¹ ${ }^{1}$.

The cells that have defective components of TGF- $B$ signaling pathway are radio-resistant. However, the cells with intact TGF- $B$ pathway tend to be show reduced TGF- $B$ response due to induction of inhibitory SMAD, SMAD7, leading to negative feedback repression of this signaling (99). SMAD7 repressor, TIEG2 (also known as KFL11) is downregulated in human cancers, inhibits cell growth in-vitro and in-vivo, and suppresses neoplastic transformation (100). Like the Smads, KLF11 is an early response transcription factor that mediates TGF-B-induced growth inhibition in untransformed epithelial cells by repressing the Smad7 expression in combination mSin3A, a corepressor (101). In our study, over expressing SMAD7 was found to make the pancreatic carcinoma cells radio-resistant (data not shown). In this context, use of TIEG2 to overcome, the SMAD7 mediated inhibition of cell death, could be an important target to enhance sensitivity to radiation through TGF-B signaling (Fig. 3).

\section{Cross-talk between hypoxia and TGF-beta signaling pathway}

Independent lines of investigation have shown that both hypoxia and $T G F-B$ can regulate the process of angiogenesis, mediated by Vascular Endothelial Growth Factor (VEGF). Sanchez et al., 2001 have demonstrated that two different signals through HIF-1 and TGF-B, synergize in stimulating VEGF transcription by functional cooperation and physical interaction (102). Similarly other genes such as erythropoietin and endoglin have also been 
reported (103, 104). However, all these studies have shown the interaction between HIF- 1 and Smads in regulating the HIF-1 specific target genes. However, very few studies show the direct effect of hypoxia on the TGF- $B$ signaling as such. In addition, how the tumor irradiation leading to re-oxygenation responds to TGF- $B$ signals need to be addressed.

\section{Summary and conclusion}

TGF- $B$ signaling is a highly complicated pathway with inputs from several different signaling pathways, genes and tumor microenvironment. Although molecular defects in TGF- $B$ signaling components such as SMAD4 and TBRII have been identified and correlated with tumor progression as well as radiation response, there are several other possible targets that need a comprehensive evaluation. An understanding of the pathway, its interaction with other players, effects of miRNAs, microenvironment in addition to the effect of radiation-induced signal transduction profile needs to be understood in order to derive a meaningful approach and to custom design gene therapeutic strategies. Findings from our group and others on the impact of TGF- $B$ signaling in the regulation of radiation response does demonstrate that restoring the function of inactivated downstream components of TGF- $B$ signaling can result in enhanced radiation sensitivity. On the contrary, completely abrogating the TGF- $B$ signaling can lead to more of a radioresistant phenotype.

\section{Acknowledgements}

This work was supported by grants from NCI (R01CA86937) and Kentucky Lung Cancer Program award to MMA.

\section{References}

1. Massague J. A very private TGF-beta receptor embrace. Mol Cell 2008;29:149-50.

2. Yang L, Moses HL. Transforming growth factor beta: tumor suppressor or promoter? Are host immune cells the answer? Cancer Res 2008;68:9107-11.

3. Petrocca F, Visone R, Onelli MR, et al. E2F1regulated microRNAs impair TGFbeta-dependent cellcycle arrest and apoptosis in gastric cancer. Cancer Cell 2008;13:272-86.

4. Martin M, Lefaix J, Delanian S. TGF-beta1 and radiation fibrosis: a master switch and a specific therapeutic target? Int J Radiat Oncol Biol Phys 2000;47:277-90.

5. Massague J. TGFbeta in Cancer. Cell 2008;134:215-30.

6. Wrzesinski SH, Wan YY, Flavell RA. Transforming growth factor-beta and the immune response: implications for anticancer therapy. Clin Cancer Res 2007;13:5262-70.
7. Annes JP, Munger JS, Rifkin DB. Making sense of latent TGFbeta activation. J Cell Sci 2003;116:217-24.

8. Annes JP, Chen Y, Munger JS, Rifkin DB. Integrin alphaVbeta6-mediated activation of latent TGF-beta requires the latent TGF-beta binding protein-1. J Cell Biol 2004;165:723-34.

9. Puthawala K, Hadjiangelis $\mathrm{N}$, Jacoby SC, et al. Inhibition of integrin alpha(v)beta6, an activator of latent transforming growth factor-beta, prevents radiationinduced lung fibrosis. Am J Respir Crit Care Med 2008;177:82-90.

10. Faresse N, Colland F, Ferrand N, Prunier C, Bourgeade MF, Atfi A. Identification of PCTA, a TGIF antagonist that promotes PML function in TGF-beta signalling. EMBO J 2008;27:1804-15.

11. Park SH. Fine tuning and cross-talking of TGF-beta signal by inhibitory Smads. J Biochem Mol Biol 2005;38:916.

12. Dennler S, Itoh S, Vivien D, ten Dijke P, Huet S, Gauthier JM. Direct binding of Smad3 and Smad4 to critical TGF beta-inducible elements in the promoter of human plasminogen activator inhibitor-type 1 gene. EMBO J 1998;17:3091-100.

13. Feng XH, Derynck R. Specificity and versatility in tgfbeta signaling through Smads. Annu Rev Cell Dev Biol 2005;21:659-93.

14. Massague J, Seoane J, Wotton D. Smad transcription factors. Genes Dev 2005;19:2783-810.

15. Feng XH, Zhang Y, Wu RY, Derynck R. The tumor suppressor Smad4/DPC4 and transcriptional adaptor $\mathrm{CBP} / \mathrm{p} 300$ are coactivators for smad3 in TGF-beta-induced transcriptional activation. Genes Dev 1998;12:2153-63.

16. Tang Y, Kitisin K, Jogunoori W, et al. Progenitor/stem cells give rise to liver cancer due to aberrant TGF-beta and IL-6 signaling. Proc Natl Acad Sci USA 2008; 105:2445-50.

17. Davis BN, Hilyard AC, Lagna G, Hata A. SMAD proteins control DROSHA-mediated microRNA maturation. Nature 2008;454:56-61.

18. Asangani IA, Rasheed SA, Nikolova DA, et al. MicroRNA-21 (miR-21) post-transcriptionally downregulates tumor suppressor $\mathrm{Pdcd} 4$ and stimulates invasion, intravasation and metastasis in colorectal cancer. Oncogene 2008;27:2128-36.

19. Frankel LB, Christoffersen NR, Jacobsen A, Lindow M, Krogh A, Lund AH. Programmed cell death 4 (PDCD4) is an important functional target of the microRNA miR-21 in breast cancer cells. J Biol Chem 2008;283:1026-33.

20. Zhu S, Wang W, Clarke DC, Liu X. Activation of Mps1 promotes transforming growth factor-beta-independent Smad signaling. J Biol Chem 2007;282:18327-38.

21. Seton-Rogers SE, Lu Y, Hines LM, et al. Cooperation of the ErbB2 receptor and transforming growth factor beta in induction of migration and invasion in mammary epithelial cells. Proc Natl Acad Sci USA 2004;101:1257-62. 22. Ueda Y, Wang S, Dumont N, Yi JY, Koh Y, Arteaga CL. Overexpression of HER2 (erbB2) in human breast epithelial cells unmasks transforming growth factor betainduced cell motility. J Biol Chem 2004;279:24505-13. 
23. Janda E, Lehmann K, Killisch I, et al. Ras and TGF[beta] cooperatively regulate epithelial cell plasticity and metastasis: dissection of Ras signaling pathways. J Cell Biol 2002;156:299-313.

24. Ventura JJ, Kennedy NJ, Flavell RA, Davis RJ. JNK regulates autocrine expression of TGF-beta1. Mol Cell 2004;15:269-78.

25. Ross KR, Corey DA, Dunn JM, Kelley TJ. SMAD3 expression is regulated by mitogen-activated protein kinase kinase-1 in epithelial and smooth muscle cells. Cell Signal 2007;19:923-31.

26. Matsuura I, Wang G, He D, Liu F. Identification and characterization of ERK MAP kinase phosphorylation sites in Smad3. Biochemistry 2005;44:12546-53.

27. Kamaraju AK, Roberts AB. Role of Rho/ROCK and p38 MAP kinase pathways in transforming growth factorbeta-mediated Smad-dependent growth inhibition of human breast carcinoma cells in vivo. J Biol Chem 2005;280:1024-36.

28. Saha D, Datta PK, Beauchamp RD. Oncogenic ras represses transforming growth factor-beta /Smad signaling by degrading tumor suppressor Smad4. J Biol Chem 2001;276:29531-7.

29. Brodin G, Ahgren A, ten Dijke P, Heldin CH, Heuchel R. Efficient TGF-beta induction of the Smad7 gene requires cooperation between AP-1, Sp1, and Smad proteins on the mouse Smad7 promoter. J Biol Chem 2000;275:29023-30.

30. Uchida K, Suzuki H, Ohashi T, Nitta K, Yumura W, Nihei H. Involvement of MAP kinase cascades in Smad7 transcriptional regulation. Biochem Biophys Res Commun 2001;289:376-81.

31. Chen RH, Chang MC, Su YH, Tsai YT, Kuo ML. Interleukin-6 inhibits transforming growth factor-betainduced apoptosis through the phosphatidylinositol 3kinase/Akt and signal transducers and activators of transcription 3 pathways. J Biol Chem 1999;274:23013-9.

32. Chen RH, Su YH, Chuang RL, Chang TY. Suppression of transforming growth factor-beta-induced apoptosis through a phosphatidylinositol 3-kinase/Aktdependent pathway. Oncogene 1998;17:1959-68.

33. Song K, Cornelius SC, Reiss M, Danielpour D. Insulin-like growth factor-I inhibits transcriptional responses of transforming growth factor-beta by phosphatidylinositol 3-kinase/Akt-dependent suppression of the activation of Smad3 but not Smad2. J Biol Chem 2003;278:38342-51.

34. Shih WL, Kuo ML, Chuang SE, Cheng AL, Doong SL. Hepatitis $\mathrm{B}$ virus $\mathrm{X}$ protein inhibits transforming growth factor-beta -induced apoptosis through the activation of phosphatidylinositol 3-kinase pathway. J Biol Chem 2000;275:25858-64.

35. Guo X, Wang XF. Signaling cross-talk between TGFbeta/BMP and other pathways. Cell Res 2009;19:71-88.

36. Yoo YA, Kang MH, Kim JS, Oh SC. Sonic hedgehog signaling promotes motility and invasiveness of gastric cancer cells through TGF-beta-mediated activation of the ALK5-Smad 3 pathway. Carcinogenesis 2008;29:480-90.
37. Zavadil J, Cermak L, Soto-Nieves N, Bottinger EP. Integration of TGF-beta/Smad and Jagged1/Notch signalling in epithelial-to-mesenchymal transition. EMBO J 2004;23:1155-65.

38. Niimi H, Pardali K, Vanlandewijck M, Heldin CH, Moustakas A. Notch signaling is necessary for epithelial growth arrest by TGF-beta. J Cell Biol 2007;176:695-707.

39. Blokzijl A, Dahlqvist C, Reissmann E, et al. Crosstalk between the Notch and TGF-beta signaling pathways mediated by interaction of the Notch intracellular domain with Smad3. J Cell Biol 2003;163:723-8.

40. Masuda S, Kumano K, Shimizu K, et al. Notch1 oncoprotein antagonizes TGF-beta/Smad-mediated cell growth suppression via sequestration of coactivator p300. Cancer Sci 2005;96:274-82.

41. Coffey RJ, Jr., Bascom CC, Sipes NJ, Graves-Deal R, Weissman BE, Moses HL. Selective inhibition of growthrelated gene expression in murine keratinocytes by transforming growth factor beta. Mol Cell Biol 1988;8:3088-93.

42. Mulder KM, Levine AE, Hernandez X, McKnight MK, Brattain DE, Brattain MG. Modulation of c-myc by transforming growth factor-beta in human colon carcinoma cells. Biochem Biophys Res Commun 1988;150:711-6.

43. Pietenpol JA, Stein RW, Moran E, et al. TGF-beta 1 inhibition of c-myc transcription and growth in keratinocytes is abrogated by viral transforming proteins with pRB binding domains. Cell 1990;61:777-85.

44. Chen CR, Kang Y, Siegel PM, Massague J. E2F4/5 and p107 as Smad cofactors linking the TGFbeta receptor to c-myc repression. Cell 2002;110:19-32.

45. Reynisdottir I, Polyak K, Iavarone A, Massague J. Kip/Cip and Ink4 Cdk inhibitors cooperate to induce cell cycle arrest in response to TGF-beta. Genes Dev 1995;9:1831-45.

46. Sandhu C, Garbe J, Bhattacharya N, et al. Transforming growth factor beta stabilizes p15INK4B protein, increases p15INK4B-cdk4 complexes, and inhibits cyclin D1-cdk4 association in human mammary epithelial cells. Mol Cell Biol 1997;17:2458-67.

47. Ahmed MM, Alcock RA, Chendil D, et al. Restoration of transforming growth factor-beta signaling enhances radiosensitivity by altering the $\mathrm{Bcl}-2 / \mathrm{Bax}$ ratio in the $\mathrm{p} 53$ mutant pancreatic cancer cell line MIA PaCa-2. J Biol Chem 2002;277:2234-46.

48. Kurokowa M, Lynch K, Podolsky DK. Effects of growth factors on an intestinal epithelial cell line: transforming growth factor beta inhibits proliferation and stimulates differentiation. Biochem Biophys Res Commun 1987;142:775-82.

49. Ko TC, Sheng HM, Reisman D, Thompson EA, Beauchamp RD. Transforming growth factor-beta 1 inhibits cyclin D1 expression in intestinal epithelial cells. Oncogene 1995;10:177-84.

50. Ko TC, Yu W, Sakai T, et al. TGF-beta1 effects on proliferation of rat intestinal epithelial cells are due to inhibition of cyclin D1 expression. Oncogene 1998;16:344554 . 
51. Sun L, Wu G, Willson JK, et al. Expression of transforming growth factor beta type II receptor leads to reduced malignancy in human breast cancer MCF- 7 cells. J Biol Chem 1994;269:26449-55.

52. Kadin ME, Cavaille-Coll MW, Gertz R, Massague J, Cheifetz S, George D. Loss of receptors for transforming growth factor beta in human T-cell malignancies. Proc Natl Acad Sci USA 1994;91:6002-6.

53. Park K, Kim SJ, Bang YJ, et al. Genetic changes in the transforming growth factor beta (TGF-beta) type II receptor gene in human gastric cancer cells: correlation with sensitivity to growth inhibition by TGF-beta. Proc Natl Acad Sci USA 1994;91:8772-6.

54. Arteaga CL, Tandon AK, Von Hoff DD, Osborne CK. Transforming growth factor beta: potential autocrine growth inhibitor of estrogen receptor-negative human breast cancer cells. Cancer Res 1988;48:3898-904.

55. Beauchamp RD, Lyons RM, Yang EY, Coffey RJ, Jr., Moses HL. Expression of and response to growth regulatory peptides by two human pancreatic carcinoma cell lines. Pancreas 1990;5:369-80.

56. Reiss M. TGF-beta and cancer. Microbes Infect 1999;1:1327-47.

57. Freeman JW, Mattingly CA, Strodel WE. Increased tumorigenicity in the human pancreatic cell line MIA $\mathrm{PaCa}^{-2}$ is associated with an aberrant regulation of an IGF-1 autocrine loop and lack of expression of the TGFbeta type RII receptor. J Cell Physiol 1995;165:155-63.

58. Inagaki M, Moustakas A, Lin HY, Lodish HF, Carr BI. Growth inhibition by transforming growth factor beta (TGF-beta) type I is restored in TGF-beta-resistant hepatoma cells after expression of TGF-beta receptor type II cDNA. Proc Natl Acad Sci USA 1993;90:5359-63.

59. Markowitz SD, Roberts AB. Tumor suppressor activity of the TGF-beta pathway in human cancers. Cytokine Growth Factor Rev 1996;7:93-102.

60. Myeroff LL, Parsons R, Kim SJ, et al. A transforming growth factor beta receptor type II gene mutation common in colon and gastric but rare in endometrial cancers with microsatellite instability. Cancer Res 1995;55:5545-7.

61. Parsons R, Myeroff LL, Liu B, et al. Microsatellite instability and mutations of the transforming growth factor beta type II receptor gene in colorectal cancer. Cancer Res 1995;55:5548-50.

62. Markowitz S, Wang J, Myeroff L, et al. Inactivation of the type II TGF-beta receptor in colon cancer cells with microsatellite instability. Science 1995;268:1336-8.

63. Garrigue-Antar L, Munoz-Antonia T, Antonia SJ, Gesmonde J, Vellucci VF, Reiss M. Missense mutations of the transforming growth factor beta type II receptor in human head and neck squamous carcinoma cells. Cancer Res 1995;55:3982-7.

64. Venkatasubbarao K, Ahmed MM, Swiderski C, et al. Novel mutations in the polyadenine tract of the transforming growth factor beta type II receptor gene are found in a subpopulation of human pancreatic adenocarcinomas. Genes Chromosomes Cancer $1998 ; 22: 138-44$.
65. Hahn SA, Schutte M, Hoque AT, et al. DPC4, a candidate tumor suppressor gene at human chromosome 18q21.1. Science 1996;271:350-3.

66. Schutte M, Hruban RH, Hedrick L, et al. DPC4 gene in various tumor types. Cancer Res 1996;56:2527-30.

67. Venkatasubbarao K, Ahmed MM, Mohiuddin M, et al. Differential expression of transforming growth factor beta receptors in human pancreatic adenocarcinoma. Anticancer Res 2000;20:43-51.

68. Friess H, Yamanaka Y, Buchler M, et al. Enhanced expression of the type II transforming growth factor beta receptor in human pancreatic cancer cells without alteration of type III receptor expression. Cancer Res 1993;53:2704-7.

69. Friess H, Lu Z, Riesle E, et al. Enhanced expression of TGF-betas and their receptors in human acute pancreatitis. Ann Surg 1998;227:95-104.

70. Bottinger EP, Jakubczak JL, Roberts IS, et al. Expression of a dominant-negative mutant TGF-beta type II receptor in transgenic mice reveals essential roles for TGF-beta in regulation of growth and differentiation in the exocrine pancreas. EMBO J 1997;16:2621-33.

71. Hahm KB, Im YH, Lee C, et al. Loss of TGF-beta signaling contributes to autoimmune pancreatitis. J Clin Invest 2000;105:1057-65.

72. Caldas C, Hahn SA, da Costa LT, et al. Frequent somatic mutations and homozygous deletions of the p16 (MTS1) gene in pancreatic adenocarcinoma. Nat Genet 1994;8:27-32.

73. Tang B, Bottinger EP, Jakowlew SB, et al. Transforming growth factor-beta1 is a new form of tumor suppressor with true haploid insufficiency. Nat Med 1998;4:802-7.

74. Yang X, Li C, Xu X, Deng C. The tumor suppressor SMAD4/DPC4 is essential for epiblast proliferation and mesoderm induction in mice. Proc Natl Acad Sci USA 1998;95:3667-72.

75. Taketo MM, Takaku K. Gastro-intestinal tumorigenesis in Smad4 mutant mice. Cytokine Growth Factor Rev 2000;11:147-57.

76. Mishra L, Cai T, Yu P, Monga SP, Mishra B. Elf3 encodes a novel 200-kD beta-spectrin: role in liver development. Oncogene 1999;18:353-64.

77. Tang Y, Katuri V, Dillner A, Mishra B, Deng CX, Mishra L. Disruption of transforming growth factor-beta signaling in ELF beta-spectrin-deficient mice. Science 2003;299:574-7.

78. Kitisin K, Ganesan N, Tang Y, et al. Disruption of transforming growth factor-beta signaling through betaspectrin ELF leads to hepatocellular cancer through cyclin D1 activation. Oncogene 2007;26:7103-10.

79. Canney PA, Dean S. Transforming growth factor beta: a promotor of late connective tissue injury following radiotherapy? Br J Radiol 1990;63:620-3.

80. O'Malley Y, Zhao W, Barcellos-Hoff MH, Robbins ME. Radiation-induced alterations in rat mesangial cell Tgfb1 and Tgfb3 gene expression are not associated with altered secretion of active Tgfb isoforms. Radiat Res 1999;152:622-8. 
81. Martin M, Vozenin MC, Gault N, Crechet F, Pfarr CM, Lefaix JL. Coactivation of AP-1 activity and TGF-beta1 gene expression in the stress response of normal skin cells to ionizing radiation. Oncogene 1997;15:981-9.

82. Anscher MS, Kong FM, Murase T, Jirtle RL. Short communication: normal tissue injury after cancer therapy is a local response exacerbated by an endocrine effect of TGF beta. Br J Radiol 1995;68:331-3.

83. Haiping Z, Takayama K, Uchino J, et al. Prevention of radiation-induced pneumonitis by recombinant adenovirus-mediated transferring of soluble TGF-beta type II receptor gene. Cancer Gene Ther 2006;13:864-72.

84. Sathishkumar S, Dey S, Meigooni AS, et al. The impact of TNF-alpha induction on therapeutic efficacy following high dose spatially fractionated (GRID) radiation. Technol Cancer Res Treat 2002;1:141-7.

85. Yang YC, Wang KL, Su TH, et al. Concurrent cisplatin-based chemoradiation for cervical carcinoma: tumor response, toxicity, and serum cytokine profiles. Cancer Invest 2006;24:390-5.

86. Satoh E, Naganuma H, Sasaki A, Nagasaka M, Ogata H, Nukui H. Effect of irradiation on transforming growth factor-beta secretion by malignant glioma cells. J Neurooncol 1997;33:195-200.

87. Reeves A, Zagurovskaya M, Gupta S, Shareef MM, Mohiuddin M, Ahmed MM. Inhibition of transforming growth factor-beta signaling in normal lung epithelial cells confers resistance to ionizing radiation. Int J Radiat Oncol Biol Phys 2007;68:187-95.

88. Wiegman EM, Blaese MA, Loeffler H, Coppes RP, Rodemann HP. TGFbeta-1 dependent fast stimulation of ATM and p53 phosphorylation following exposure to ionizing radiation does not involve TGFbeta-receptor I signalling. Radiother Oncol 2007;83:289-95.

89. Portess DI, Bauer G, Hill MA, O'Neill P. Low-dose irradiation of nontransformed cells stimulates the selective removal of precancerous cells via intercellular induction of apoptosis. Cancer Res 2007;67:1246-53.

90. Chorna I, Fedorenko O, Datsyuk L, Stoika R. Expression of mRNA coding for TGF-beta and its receptors in irradiated human breast carcinoma $\mathrm{MCF}-7$ cells differing in their sensitivity to doxorubicin. Exp Oncol 2005;27:156-8.

91. Bruyneel EA, Storme GA, Schallier DC, Van den Berge DL, Hilgard P, Mareel MM. Evidence for abrogation of oncogene-induced radioresistance of mammary cancer cells by hexadecylphosphocholine in vitro. Eur J Cancer 1993;29A:1958-63.

92. Hermens AF, Bentvelzen PA. Influence of the H-ras oncogene on radiation responses of a rat rhabdomyosarcoma cell line. Cancer Res 1992;52:3073-82.

93. Miller AC, Gafner J, Clark EP, Samid D. Differences in radiation-induced micronuclei yields of human cells: influence of ras gene expression and protein localization. Int J Radiat Biol 1993;64:547-54.

94. Miller AC, Kariko K, Myers CE, Clark EP, Samid D. Increased radioresistance of EJras-transformed human osteosarcoma cells and its modulation by lovastatin, an inhibitor of p21ras isoprenylation. Int $J$ Cancer 1993;53:302-7.

95. Calonge MJ, Massague J. Smad4/DPC4 silencing and hyperactive Ras jointly disrupt transforming growth factor-beta antiproliferative responses in colon cancer cells. J Biol Chem 1999;274:33637-43.

96. Song SY, Meszoely IM, Coffey RJ, Pietenpol JA, Leach SD. K-Ras-independent effects of the farnesyl transferase inhibitor L-744,832 on cyclin B1/Cdc2 kinase activity, G2/M cell cycle progression and apoptosis in human pancreatic ductal adenocarcinoma cells. Neoplasia 2000;2:261-72.

97. Alcock RA, Dey S, Chendil D, et al. Farnesyltransferase inhibitor (L-744,832) restores TGFbeta type II receptor expression and enhances radiation sensitivity in $\mathrm{K}$-ras mutant pancreatic cancer cell line MIA PaCa-2. Oncogene 2002;21:7883-90.

98. el-Deiry WS, Harper JW, O'Connor PM, et al. WAF1/CIP1 is induced in p53-mediated G1 arrest and apoptosis. Cancer Res 1994;54:1169-74.

99. Segreto HR, Ferreira AT, Kimura ET, et al. Amifostine does not prevent activation of TGFbeta1 but induces smad 7 activation in megakaryocytes irradiated in vivo. Am J Hematol 2002;71:143-51.

100.Fernandez-Zapico ME, Mladek A, Ellenrieder V, Folch-Puy E, Miller L, Urrutia R. An mSin3A interaction domain links the transcriptional activity of KLF11 with its role in growth regulation. EMBO J 2003;22:4748-58.

101.Ellenrieder V, Buck A, Harth A, et al. KLF11 mediates a critical mechanism in TGF-beta signaling that is inactivated by Erk-MAPK in pancreatic cancer cells. Gastroenterology 2004;127:607-20.

102.Sanchez-Elsner T, Botella LM, Velasco B, Corbi A, Attisano L, Bernabeu C. Synergistic cooperation between hypoxia and transforming growth factor-beta pathways on human vascular endothelial growth factor gene expression. J Biol Chem 2001;276:38527-35.

103. Sanchez-Elsner T, Botella LM, Velasco B, Langa C, Bernabeu C. Endoglin expression is regulated by transcriptional cooperation between the hypoxia and transforming growth factor-beta pathways. J Biol Chem 2002;277:43799-808.

104.Sanchez-Elsner T, Ramirez JR, Sanz-Rodriguez F, Varela E, Bernabeu C, Botella LM. A cross-talk between hypoxia and TGF-beta orchestrates erythropoietin gene regulation through SP1 and Smads. J Mol Biol 2004;336:924. 